\title{
A Genetic Algorithm Approach to Optimization of Power Peaks in an Automated Warehouse
}

\author{
J. J. Cárdenas, A. Garcia, J. L. Romeral, F. Andrade \\ Motion Control and Industrial Applications Group, Technical University of Catalonia, \\ C/ Colon 1 Tr 2-225. 08222 Terrassa. Catalonia. Spain, e-mail: romeral@,eel.upc.edu
}

\begin{abstract}
The simultaneous operation of the automated storage and retrieval machines (ASRs) in an automated warehouse can increase the likelihood that high power demand peaks turn unstable the electric system. Furthermore, high power peaks mean the need for more electrical power contracted, which in turns leads to more fixed operation cost and inefficient use of the electrical installations. In this context, we present a genetic algorithm approach to implement demandside management (DSM) in an automated warehouse. It has been based on real data from ASRs and models of prognosis of load profile of ASRs. We took into account two main goals: minimize instantaneous power demand and keeping the performance of the system store and retrieval times.
\end{abstract}

Key words - Genetic algorithm, demand-side management, automated warehouse, storage and retrieval machines.

\section{INTRODUCTION}

Warehouse is a facility, which provides the services about material storage and management to a manufacturing firm or customer. Its efficiency is depended on many factors and it is important because costs incurred are reflected in the production or distribution accounts, and are ultimately on to the consumer [1]. Generally speaking, the efficiency of warehouse operations is influenced by many factors such as warehouse layout, storage policy, order picking policy, etc. [2].

On other hand, it is observed that the simultaneous operation of the automated storage and retrieval machines (ASRs) in an automated warehouse can increase the likelihood that high power demand peaks turn unstable the electric system. Apart from instability, these peaks cause the deterioration of the power quality, increasing the dips and swells events, even operation stops caused for power cuts. In general, to avoid this the factories oversize their electrical installations, so that the probability of this happening is very low. Oversizing is to use the cable with a caliber greater than necessary, having greater electrical cabinets and boxes, as well as generators and transformers have a higher electrical power capacity. Of course, this increases significantly the cost of building an automated warehouse and the problems about power quality remain. Furthermore, it is usual that the utilities charge in the electrical bill the contracted power, which is the power that the utilities foresee available for a specify costumer. If the costumer over pass this power it has to pay penalties, which increase the electrical bill.

Hence, making efficient use of electricity is as important as the optimization of any other resource or raw material.
Therefore, it is essential to look for methods or algorithms in order to improve the energy use efficiency, even when we have to decrease a little the performance of other parameters.

Nowadays, at scientific literature we can find subjects related with automated warehouse, focusing in problems like optimization of the best route of the ASRs for minimizing the storage and retrieval times, and making decisions to establishing which it is the best way to arrange the goods in the warehouse. All these subjects are important in order to reduce the operating time and improve the efficiency of the system [3-6]. However, the simultaneous problem of power peaks in factory installations has not been taken into account directly in the scientific literature.

A simply solution in order to improve the energy used could be arranging the start of the ASRs so that the simultaneous startups are avoided. It could be useful since the peak power of rotating machines generally happen at the beginning of the movement. However, this solution has several buts: the peaks not always appear at the beginning, and there are peaks at the end or in the middle of the movement. Also, the length of the peaks is variable and it depends on random variables as the weight of the load, the distance to the goal place where the load will be stored and the start point, the velocity of the ASRs in x-axis as y-axis, etc. This shows clearly that the problem is not trivial and it is not deterministic for its random nature and it requires of a dynamic algorithm. So stochastic methods are naturally selected for modeling and optimization solution of such kind of problem [7, 8]. This is the main reason because the use of Genetic Algorithms (GA) as first approach to search for the optimal timing distribution of ASRs.

This approach looks for the best using of available power, improving the electrical stability, decreasing the fixed cost by contracted power and allowing the best uses of electrical installations, which decreases the electrical building costs.

With a good string coding of space of search it is possible to implement a system with such characteristics and furthermore it is able to work fast and it does not decrease the performance of operation of the whole storage system.

First in the paper, we present the modeling of ASRs that is indispensable for the application. This is presented in the Section II. In the Section III we explain how deal with the problem using a GA and introduce the main concepts about this evolutionary algorithm. Section IV presents the got simulation results and finally Section $\mathrm{V}$ draws some conclusions and further work. 


\section{LOAD PROfILE MOdeLING OF ASRS}

For this Section we have extracted from the real data a model that is easy to use and it requires a few resources to its management. The data could also be extracted from a prognosis module of load profiles. Therefore, we have used the real data only to get the models, because we suppose that in a full application we would have a prognosis module of load profiles, which would supply the load profile with the shape of the proposed models. This hypothetical prognosis module has as main inputs the allocation coordinates $[\mathrm{X}, \mathrm{Y}]$ from optimization allocation module. It would be expected that such load profile prognosis is not so accurate and detailed for a load profile with minutes' resolution. Despite this, the most important is that the general algorithm for DSM does not ask for an accuracy prognosis of a load profile. However, it is required that the prognosis shows the shape of the load profile and especially indicates with a good enough degree of accuracy and reliability of the occurrence of power peak demand.

The measurements of currents were made with and without load on the ASRs. The selected load profiles (Fig. 1, Fig. 2 and Fig. 3) show clearly the main characteristics of each one of the movements. It was detected a strong dependency among them and the input variables $\mathrm{X}, \mathrm{Y}$ (Cartesian coordinates of the positions in the warehouse) and the load weight. However, the form of each profile in general is mainly dependent of the kind of movement to execute (translation on the $\mathrm{x}$-axis in forward and reverse movement, elevation or descent on the y-axis). As expected, the load weight and $\mathrm{X}, \mathrm{Y}$ position influence mainly the maximum peaks and the duration of the profiles.

Fig. 1 is a clear example of the simplicity of the used models. We can see in red, the real Load Profile (LP) of some tested ASRs and in black, the proposed model. This LP is for a particular movement, in this case the translation on $\mathrm{x}$ axis (as it was explained former).

Only five points characterize the model proposed for this movement, and only three of them are representatives. The others are the initial and the final points. These points are:

Init point: $\left(I_{\text {ini }}, t_{l x}\right)$; $\quad$ Maximun peak: $\left(I_{\max }, t_{\text {sul }}\right)$

Nominal current: $\left(I_{n}, t_{b a l}\right)$; Down point: $\left(I_{n}, t_{n}\right)$;

Final point: $\left(I_{f i n}, t_{b a 2}\right)$

For the others movements (up and down) the modeling are similar, the only different is the amount of parameters because as we said before the LP is depend about the kind of movement.

In the Fig. 2, again, we have in red the real data and in black the proposed model for the up movement. In this case, the parameters of the model are:

Init point: $\left(I_{\text {ini }}, t_{l y}\right) ; \quad$ Nominal current: $\left(I_{n}, t_{\text {sul }}\right)$;

Down point: $\left(I_{n}, t_{n}\right) ; \quad$ Final point: $\left(I_{\text {fin }}, t_{\text {bal }}\right)$

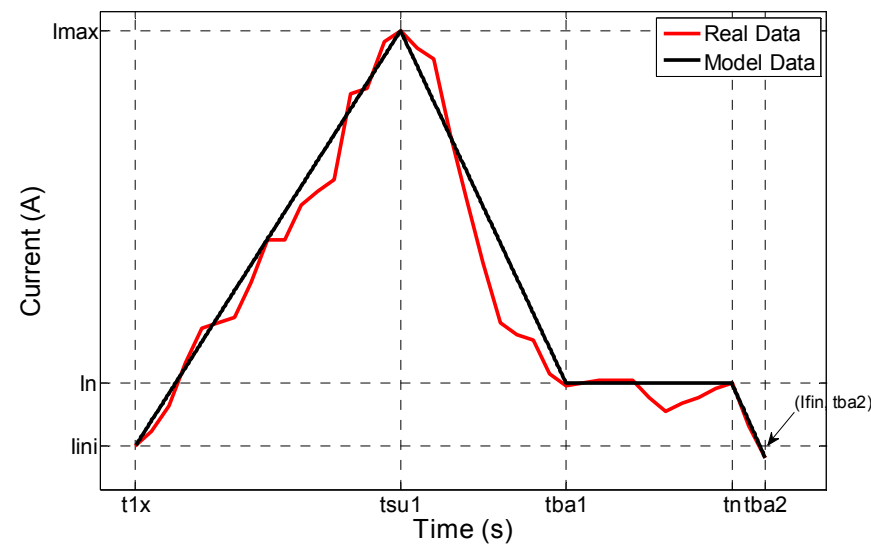

Fig. 1. The load profile of an ASR without load in forward movement on the $\mathrm{x}$-axis. In red color the real load profile and in black color the load profile model.

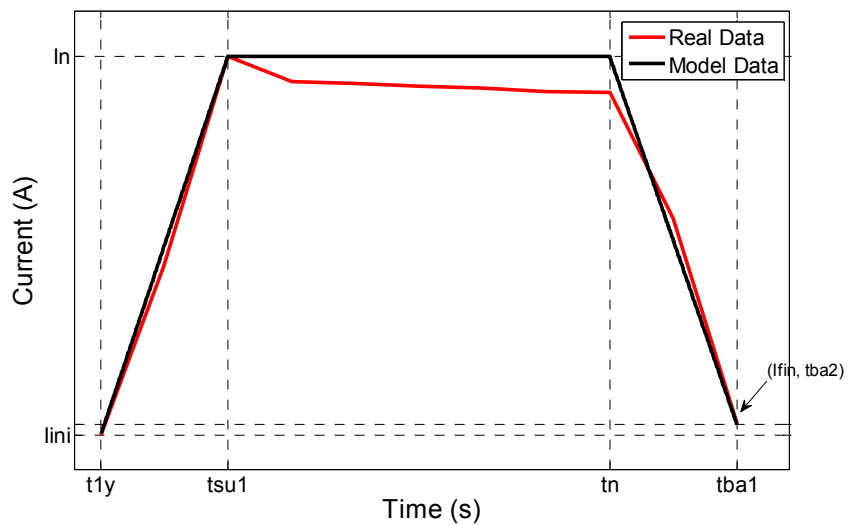

Fig. 2. The load profile of an ASR without load in up movement. In red color the real load profile and in black color the load profile model.

Finally, in the Fig. 3 we have the model and real data for the down movement. The main parameters are:

Init point: $\left(I_{\text {ini }}, t_{2 y}\right) ; \quad$ Current first peak: $\left(I_{\text {peak }}, t_{\text {sul }}\right)$

Nominal current: $\left(I_{n}, t_{b a l}\right)$ to $\left(I_{n}, t_{n}\right)$;

Current second peak: $\left(I_{\text {peak }}, t_{\text {su } 2}\right)$; Final point: $\left(I_{\text {fin }}, t_{\text {ba2 }}\right)$

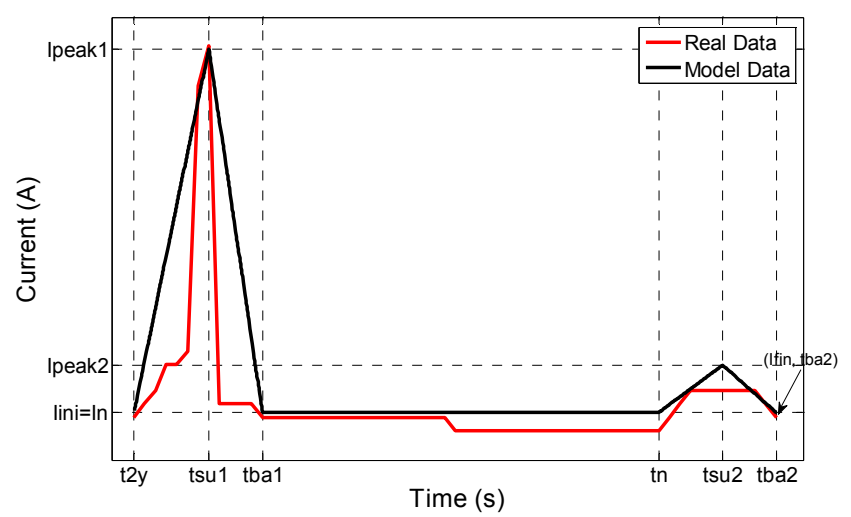

Fig. 3. The load profile of an ASR without load in down movement. In red color the real load profile and in black color the load profile model. 
Each of these movements is related to its corresponding motor on the ASR. It has a motor for movement in each axis, hence the LP in Fig. 1 corresponds to the motor for movement in $\mathrm{x}$-axis (horizontal translation) and the LP in Fig. 2 and Fig. 3 correspond to the motor of the movement in y-axis (up and down). Therefore, to get the total LP of a storage process (round trip) we have superimposed the corresponding LPs depending on their temporal location. In the Fig. 4 is showed the LP for a full movement of storage below to an ASR. The LP is divided in two parts: at the top is the LP of the axis-x movement and the bottom is the LP of the axis-y movement.

Fig. 4 shows how the timeline is segmented for each of the steps performed by the ASR in a storage movement. $t_{1 x}$ and $t_{1 y}$ are the start times. So $\Delta t_{1 x}$ and $\Delta t_{1 y}$ are the used delays to avoid the simultaneous start of the motors among those belong to the same ASR and among the motors of the different ASRs in the warehouse. The translation movement with load is made between $T_{x}$ and $t_{1 x}$ times; the up movement with load, between $T_{y}$ and $t_{1 y}$ times; so far as the going movements are executed, then the going time is determinate for the longest between $T_{x}$ and $T_{y}$. When $[\mathrm{X}, \mathrm{Y}]$ position are reached the fork movement is executed. This is to leave the load on its storage position and it takes the time interval between $t_{f}$ and the final going time ( $T_{x}$ or $T_{y}$ as appropriate). Then, the ASR has to return to the starting position and pick up the next load. The return without load movement has the following sequence: again there are delay times to avoid simultaneous start and these are $\Delta t_{2 x}$ and $\Delta t_{2 y}$; the return horizontal and the down movements are executed between $T_{x \text { total }}$ and $t_{2 x}, T_{y \text { total }}$ and $t_{2 y}$ times, respectively. The total time of the whole movement will be the maxim between $T_{x t o t a l}$ and $T_{y \text { total }}$.

It has been show the modeling of the load profiles, and it is important highlight their simplicity and practicality, which make it suitable to be implemented for a prognosis algorithm, for example, mean artificial neuronal networks.
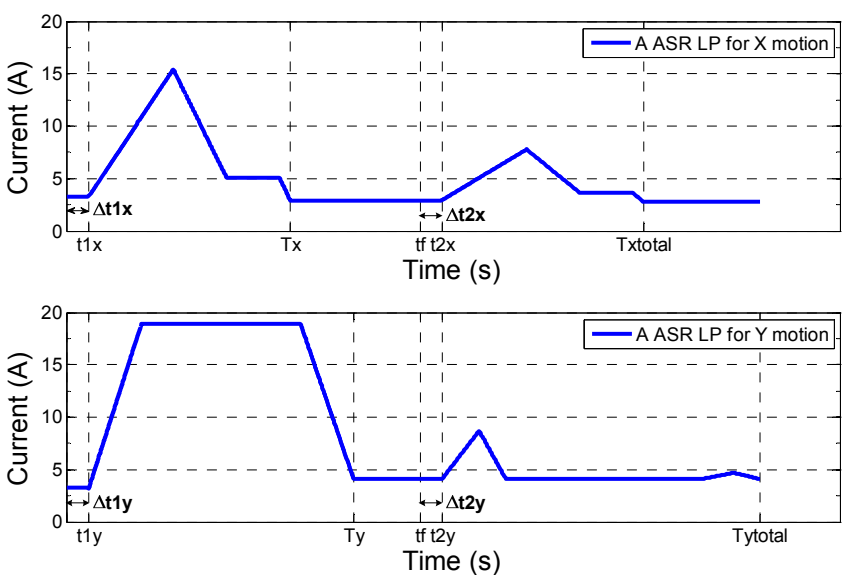

Fig. 4. Full LP for an ASR. Fig. 4a. At the top is the LP for $x$-axis movements (x motor of the ASR). Fig. 4b. LP for $y$-axis movements (y motor of the ASR)

\section{GENETIC Algorithm SOLUTION}

The proposed solution based in a Genetic Algorithm (GA) [9] to get the optimal delay times $\left(\Delta t_{1 x}, \Delta t_{1 y}, \Delta t_{2 x}, \Delta t_{2 y}\right)$ for each ASR in the warehouse, is presented in the Fig. 5 as a general block diagram. The first block has as inputs the $[\mathrm{X}$, Y] coordinates, which determine the position where the objects have to be stored or retrieved. This block is used to get the LPs prognosis. The next block is the GA optimization, where the GA is executed and it has as outputs the times delays that optimize the use of the available electric power. The final block is only used to visualize the results and do the comparison between the init and final data.

For this first implementation we have only had into account two ASRs, which are named A and B. The aim of this is to simplify the development. However, to add a new ASR or modify the application so that the number of ASRs will be a parameter, it is not hard and there are no constraints relative to the GA implementation. The having more ASRs or machines to management mainly affect the convergence time of the GA. This was tested in another application where fictitious load profiles were used to simulate the management of multiples machines (for example, more than 10 machines). Although the convergence time was increased, the generation's number remains about constant and such increase it was not so much in comparison with simulations with a low number of LPs (for example, less than 10 machines)

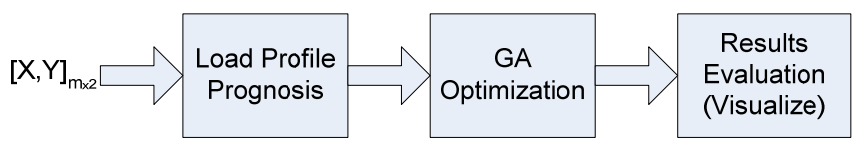

Fig. 5. The general block diagram of the proposed solution.

\section{A. LP Prognosis}

Taking advantage of the modeling simplicity here presented, an algorithm to do the prognosis of the load profile in function of the $[\mathrm{X}, \mathrm{Y}]$ coordinates could easily be implemented, such as an algorithm with artificial neural networks. Now, we have used the directly got models from the real data as we explained in the "Load Profile modeling of ASRs" section.

In order to implement this module, we divided it in two parts, as showed in the Fig. 6: The "Get Model Parameters" and the "Make Load Profile" sub models. The first has the task of getting the main parameters of each LP, as showed in the modeling section. Now it is made of semi-automated way. The second sub model uses the obtained parameter to concatenate the vector that represents each LP. These vectors are A.lp and B.lp and others parameters that are encapsulated in $A$ and $B$ structures.

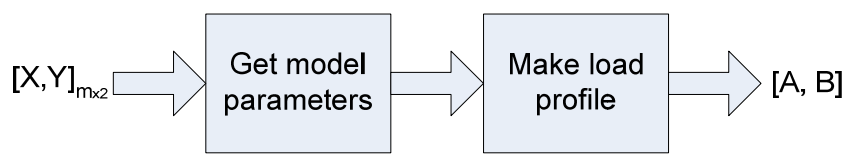

Fig. 6. Block diagram of the prognosis module. 


\section{B. GA solution}

GA consists on searching algorithms based on the mechanics of natural selection and natural genetics. They combine survival of the fittest among string structures (chromosomes) with a structures yet randomized information exchange to form a search algorithm with some of the innovative flair of human search. In every generation, a new set of artificial chromosomes (strings) are created using pieces of the fittest of the old; an occasional new part is tried for good measure; these new chromosomes are gotten means functions or operators that mainly emulate the evolutionary processes of selection, mating and mutation. While randomized, genetic algorithms are no simple random walk, they efficiently exploit historical information to speculate on new search points with expected improved performance. These algorithms are computationally simple yet powerful in their search for improvement. Furthermore, they are not fundamentally limited by restrictive assumptions about the search space (assumptions concerning continuity, existence of derivatives, unimodality, and other matters) Genetic Algorithms have been developed by John Holland, his collogues, and his students at the University of Michigan [9].

The Fig. 7 shows the flowchart of a standard GA. Firstly, the initial population is obtained by means of random initialization of the each chromosome of the population. The number of individuals or chromosomes is a very important parameter of the GA. So the diversity of the population depends on population size and that guarantees the no getting into local minimum. Nevertheless, a very large population could make so slow the execution of the GA. For this application, we chose a population initial of 20 individuals.

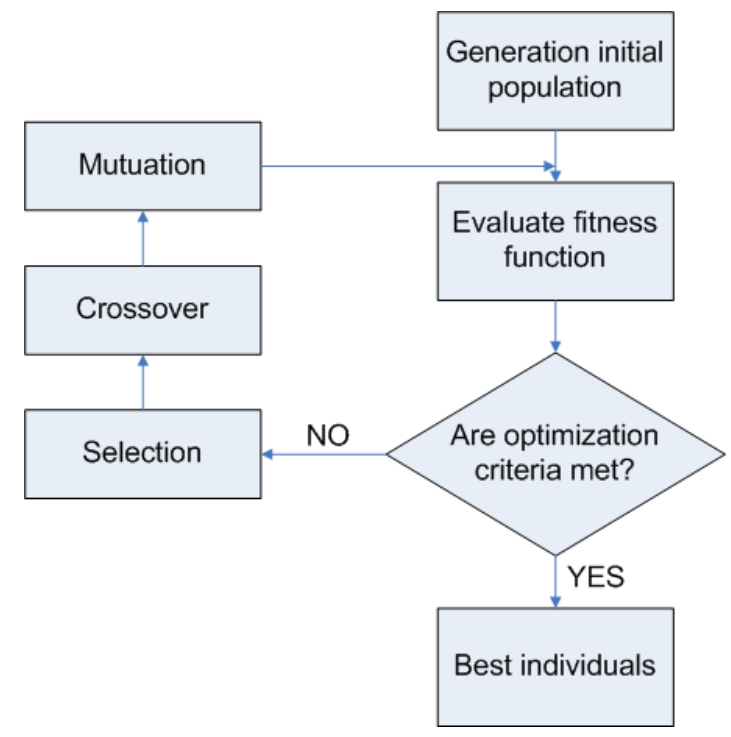

Fig. 7. Flowchart of a GA.

Next it is executed the calculation of the fitness function. This is the function objective, which has to be minimized. In this case, we want to minimize the power peaks of the total load profile. Therefore, the fitness function is determinate by means of the next equations:

$t$ : time. $k$ : number of the actual iteration.

$M$ : number of ASRs.

$f v$ : value of the fitness function for the iteration $k$.

$P_{k, \text { max }}:$ max power peak for the iteration $k$.

$l p(t)_{k, i}$ : the load profile of the ASR $i$, in the iteration $k$.

$x$ : vector of time delays that determinate the sequence of start of the ASRs.

$$
\begin{gathered}
f v_{k}=P_{k, \max }=\max \left(\sum_{i=1}^{M} l p(t)_{k, i}\right) \\
\min _{x} P_{k, \max }
\end{gathered}
$$

Then the equation (2) is the target of the GA and the $x$ vector is formed for the time delays that determinate the start of the ASRs as it is showed in the Fig. 4. Thus, the immediate coding of the chromosome of the GA is the showed in the Fig. 8. The first 4 gens belong to the A ASR and the next 4 to the B ASR.

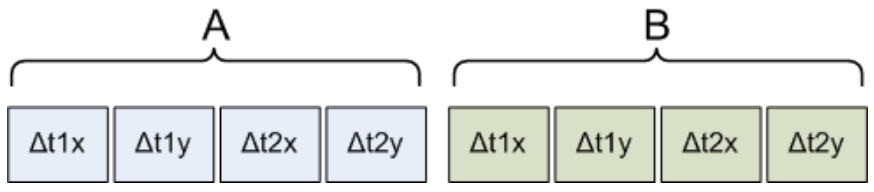

Fig. 8. String coding for the GA of the proposed solution.

The next step of the GA is the evaluations of the optimization criteria. If any are met, then the GA is stopped, or else, the evolutionary operators are used in order to get the next generation.

The used operators in this application were the selection, crossover and mutation ones. As its name suggests the selection consists in to select the best individuals from actual population. There are many ways to execute this operator [9]. Here we pass them to the next generation directly. The number of selected individuals is another parameter of the GA and determinates the degree of opportunity reproduction of the best individuals. This is named the selective pressure. The crossover or mating is the process of crossing over the genetic material from the parents to create the genetic material of the children. The crossover can be done of different ways. In this case, we have used the scattered function, which takes the genetic material from two parents and crosses over them following a generated random binary vector. Where the binary vector is 1 the gens are taken from parent 1 and in another case from parent 2. For example, if $p 1$ and $p 2$ are the parents

$$
\begin{aligned}
& p 1=\left[\begin{array}{llll}
a & b c d e f g h
\end{array}\right] \\
& p 2=\left[\begin{array}{lllllll}
1 & 2 & 3 & 4 & 5 & 6 & 7
\end{array}\right]
\end{aligned}
$$

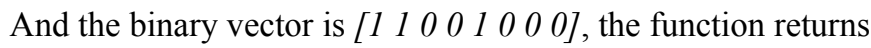
the following child: 


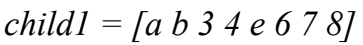

Mutation operation applies random changes to individual parents to form children. Mutation provides genetic diversity and enables the genetic algorithm to search a broader space. We have used Gaussian mutation, which adds a random number taken from a Gaussian distribution with mean 0 to each entry of the parent vector.

In summary, the final settings of the GA used were:

- Initial population: 20 random generated individuals

- Next generation: 2 selection +16 crossover +2 mutation

- Selection: stochastic uniform.

- Crossover: scattered

- Mutation: Gaussian

The software of simulation used was Matlab ${ }^{\circledR}$ which has a toolbox of Genetic Algorithm and Direct Search.

Next, we continue with the simulation results that were got by means of this configuration of the GA first approach solution.

\section{Simulation Results}

In the Fig. 9 is presented the simulation result. The initial load profiles are at the top plot and final load profiles at the bottom plot. In both, the load profile of $A, B$ and the total is in blue, red and black respectively. With a simple inspection, we can notice the differences in terms of duration, appearance and peak profiles. In the initial load profiles, we have a maxim peak of about 56 A that occurs around the 4 seconds. This peak is generated by the maxim peak of $A$ and the rising load profile of $B$. Remember that each load profile is built by the addition of the load profile of each motor in the same ASR and the total load profile by the addition of the two ASRs under test, $A$ and $B$. These initial profiles are the supposed prognostic load profiles. At the bottom are the final load profiles after the GA execution and reordered the start times of each motor in both ASRs. There we can see as they have changed. Now the maxim peak on the total load profile is $46 \mathrm{~A}$ at the $5.5 \mathrm{~s}$ time. It has been reduced in $21 \%$, which is an appreciable result. However, also it is important to remark that the total time has been increased too. The initial total time was about $29 \mathrm{~s}$ and after the rearrangement, this was about $34 \mathrm{~s}, 5 \mathrm{~s}$ more than initial time. This is because the maxim delay time allowed was of $5 \mathrm{~s}$, which is specified as a constraint in the GA settings.

In Fig. 10 shows the rapid convergence of GA for this particular application. There we can see that about the $40^{\text {th }}$ iteration the population converge and the $51^{\text {th }}$ iteration the GA stop because some stop criteria is met. This is the average change in the fitness function value less than the threshold settings.
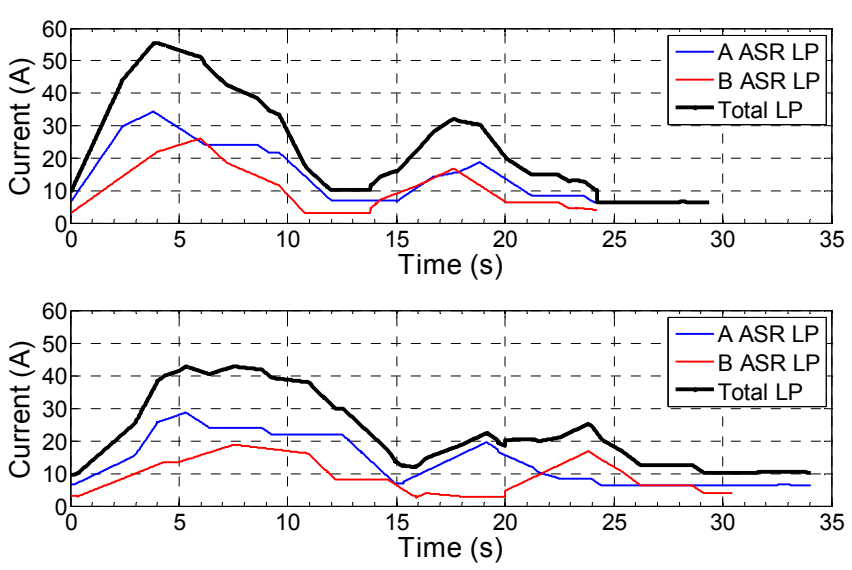

Fig. 9. Initial (at the top) and final (at the bottom) LP for the A, B ASRs and the total LP. In black color the total LP, in blue color the A ASR LP and in red color the B ASR LP.

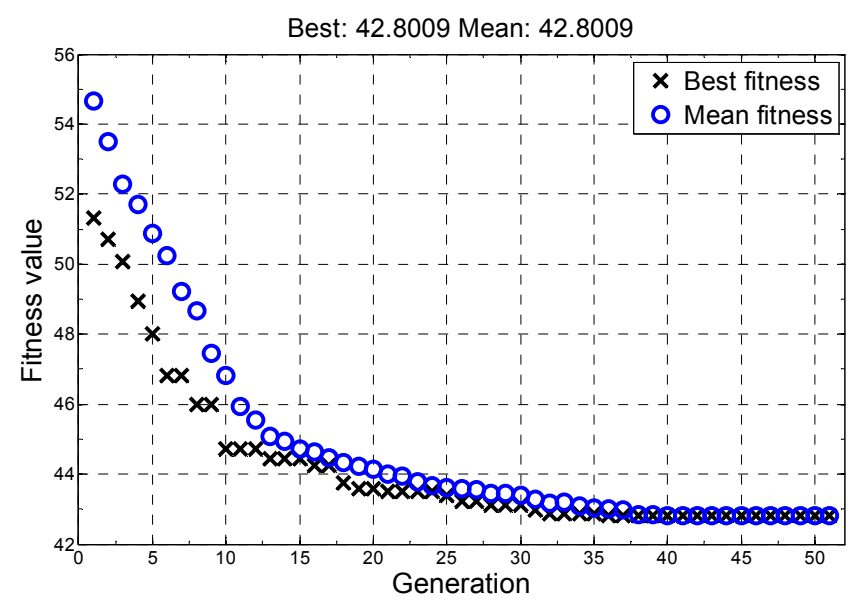

Fig. 10. Population evolution of the GA.

The Fig. 11 shows the best individual obtained by the genetic algorithm. Comparing this figure with the coding figure (Fig. 8) and the building structure of the load profile of an ASR (Fig. 4), we see that the maxim delay is in the start time of the $B$ ASR on $\mathrm{x}$-axis (or $x$ motor) The next long delay is the start time of the $y$ motor of the $A$ ASR. Both on the going movement.

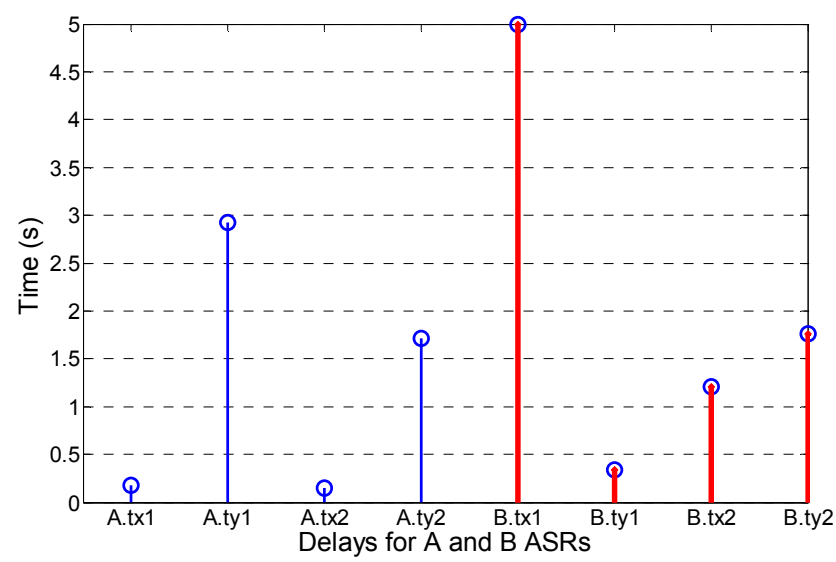

Fig. 11. The best individual. 
Finally, the TABLE I shows a summary of the obtained results. There we can see that the maximum peak for the processed profile was 44 Amps, which is $21 \%$ less than initial load peak. However, the total time is increased in 5 seconds, which is the $17 \%$ of the initial total time. The maximum allowed delay or the ratio between this and the decrease of the maximum load peak depend on the application and customer needs.

TABLE I

SUMMARY RESULTS FOR THE IMPLEMENTED GA

\begin{tabular}{l|ccc}
\hline \multicolumn{1}{c|}{ Description } & Value & Unit & Observation \\
\hline ASR number & 2 & uni & A and B \\
Motors for control & 2 & uni & x and y axis \\
Total searched delays & 8 & uni & 4 by ASR \\
Allowed maximum delay & 5 & s & \\
Maximum obtained delay & 5 & s & \\
Initial total time & 29 & s & \\
End total time & 34 & S & \\
Initial maximum total load peak & 56 & A & \\
Final maximum total load peak & 44 & A & \\
Load peak reduction & $21 \%$ & $\%$ & Ratio between \\
\hline
\end{tabular}

\section{CONCLUSIONS}

It is important to notice that energy optimization issues are raising more and more due to the current worldwide energetic problem.

A GA approach for solving the sequencing problem to get optimal use of available power in an automated warehouse was implemented with appreciable results regarding the reduction of the instantaneous maxim power demand.

This shows the feasibility and the potential of this kind of algorithm to solve problems of DSM in automated warehouse.

To develop a full DSM application, the next step is to implement a prognosis algorithm to get the load profile prognosis. This algorithm could be a neural network in order to take advantage of the method of modeling proposed here. Therefore, the system will be able to be tested in a real application.

\section{REFERENCES}

[1] J. Drury and P. Falconer, Building and planning for industrial storage and distribution, 2 ed.: Architectural Press, 2003.

[2] S. Chwen-Tzeng, "Intelligent control mechanism of part picking operations of automated warehouse," in Industrial Automation and Control: Emerging Technologies, 1995., International IEEE/IAS Conference on, 1995, pp. 256-261.
[3] S.-n. Liu, "Optimization problem for AGV in automated warehouse system," in Service Operations and Logistics, and Informatics, 2008. IEEE/SOLI 2008. IEEE International Conference on, 2008, pp. 1640-1642.

[4] C. Yueting and H. Fang, "Research on particle swarm optimization in location assignment optimization," in Intelligent Control and Automation, 2008. WCICA 2008. 7th World Congress on, 2008, pp. 111-116.

[5] L. Meijuan, C. Xuebo, and Z. Meifeng, "Optimal Scheduling Approach of Storage/Retrieval Equipments Based on Genetic Algorithm," in Intelligent Control and Automation, 2006. WCICA 2006. The Sixth World Congress on, 2006, pp. 33453348.

[6] L. Meijuan and C. Xuebo, "Research on optimization problem of the automated warehouse using an improved ant colony algorithm," in Intelligent Control and Automation, 2008. WCICA 2008. 7th World Congress on, 2008, pp. 8716-8721.

[7] L. Y. Seng and P. Taylor, "Innovative Application of Demand Side Management to Power Systems," in Industrial and Information Systems, First International Conference on, 2006, pp. 185-189.

[8] Y. K. Penya., "Last-generation Applied Artificial Intelligence for Energy Management in Building Automation," in In the Proceedings of the 5th IFAC International Conference on Fieldbus Systems and their Applications, FeT 2003 Aveiro Portugal, 2003.

[9] D. E. Goldberg, Genetic Algorithms in Search, Optimization, and Machine Learning: AddisonWesley Longman, Inc., 1989. 